\title{
Functional Massage of the Teres Major Muscle in Patients with Subacromial Impingement Syndrome. A Randomized Controlled Case Series Study
}

\author{
Martín Eusebio Barra-López $z^{1,2,3^{*}}$, María Orosia Lucha-López ${ }^{4}$, \\ Sara Castillo-Tomás ${ }^{5}$, Carlos López-de-Celis ${ }^{1,2,3}$, Vanessa González-Rueda ${ }^{1,2}$, \\ Edurne Villar-Mateo ${ }^{1,2}$ and Ana Domínguez-Cobo ${ }^{1}$
}

${ }^{1}$ Rehabilitation Service Baix Llobregat Centre, DAP Costa de Ponent, Catalan Institute of Health, Barcelona, Spain.

${ }^{2}$ Jordi Gol Institute of Research on Primary Health Care, Barcelona, Spain. ${ }^{3}$ Faculty of Medicine and Health Sciences, International University of Catalonia, Barcelona, Spain.

${ }^{4}$ Faculty of Health Sciences, University of Zaragoza, Zaragoza, Spain.

${ }^{5}$ FREMAP Mutual Society for Work-related Injuries and Occupational Illness, Arnedo (La Rioja),

Spain.

\section{Authors' contributions}

This work was carried out in collaboration between all authors. Author MEBL designed the trial, analysed data and wrote the draft of the manuscript. Author MOLL supervised the work. Author SCT

contributed to the design of the trial and provided subjects. Author CLC designed the figures and contributed to the correction of the draft. Author VGR provided subjects and contributed to the correction of the draft. Author EVM contributed to provide subjects and contributed to the correction of the draft. Author ADC managed literature searches. All authors read and approved the final

manuscript.

Article Information

DOI: $10.9734 /$ IJMPCR/2016/29294

Editor(s):

(1) José M. Matés, Department of Molecular Biology and Biochemistry, Faculty of Sciences, University of Málaga, Spain. Reviewers:

(1) Olufemi O. Oyewole, Olabisi Onabanjo University Teaching Hospital, Sagamu, Nigeria. (2) Terry J. Ellapen, University of KwaZulu-Natal, South Africa. (3) Timothy Hui, Loma Linda University, CA, USA. Complete Peer review History: http://www.sciencedomain.org/review-history/16572 


\section{ABSTRACT}

Aims: Subacromial impingement syndrome is the most common shoulder condition. Myofascial trigger points in teres major muscle can be associated with this syndrome. Our objective is to determine whether adding manual therapy specifically for teres major trigger points can produce better results in these patients.

Study Design: Randomized controlled case series.

Place and Duration of Study: Public Primary Care Center in the Spanish National Health System (Cornellà de Llobregat - Barcelona) and the FREMAP Mutual Society for Work-related Injuries and Occupational Illness (Arnedo - La Rioja), between January and March 2014.

Methodology: Fifty-eight people were recruited but 8 subjects were lost during the follow-up period. The sample consisted of 50 patients (17 male and 33 female, age range 23-80 years) randomly assigned to one of two groups: the intervention group or the control group. Both groups received a protocolized physical therapy treatment, while the intervention group also received manual therapy for teres major trigger points.

Results: Pain intensity $(p=.01)$ and function $(p=.01)$ showed significant improvement in the control group, whereas pain intensity $(p=.01)$, function $(p=.01)$ and active range of motion $(p=.01)$ showed significant improvement in the intervention group. Between-group differences were statistically significant for abduction $(p=.01)$, extension $(p=.02)$ and lateral rotation $(p=.02)$, and clinically significant (Cohen's $d$ ) for function, flexion, extension, lateral rotation and abduction.

Conclusion: Although our findings must be considered as preliminary, they suggest that adding manual therapy to treat teres major trigger points achieves better results in the glenohumeral range of motion.

Keywords: Functional massage; subacromial impingement syndrome; manual therapy; teres major muscle; physical therapy.

\section{INTRODUCTION}

The prevalence of shoulder pathology ranges between $16 \%$ in the general population [1] and $21 \%$ in the population over 70 years old [2]. In the Spanish population of working age, the shoulder is the extremity region with the highest percentage of subjects affected by musculoskeletal symptoms (13.8\%), only exceeded by the lumbar (44.9\%), cervical (34.3\%) and dorsal (27.1\%) spines [3]. The incidence has been estimated at 11.2 per 1,000 patients/year, with a majority of cases (41\%) diagnosed with subacromial impingement syndrome (SIS) [4]. SIS is characterized by pain emanating from subacromial space structures that increases with upper extremity elevation, and restriction of mobility causing functional limitation affecting the patient's quality of life [5].

A biomechanical cause that can cause the impingement of the subacromial structures is the lack of coordination of muscle activation during extremity elevation [6]. Most studies of muscle coordination have been based on the model of Inman et al. [7] which focuses on the role of the infraspinatus, teres minor and subscapularis muscles opposing the deltoid muscle in order to minimize the impact of the humeral head against the coracoacromial arch during elevation. However, a recent study has included the evaluation of other adductor muscles, considering the classic concept of normal function of the shoulder obtained by a balance between the deltoid and rotator cuff muscles as inadequate [8].

In a study evaluating muscle activity during a functional elevation and depression movement of the extremity, Hawkes et al demonstrated that the teres major muscle is more active during elevation than during depression in asymptomatic subjects. Moreover, during the elevation phase, the maximal activity peak of the adductor group (latissimus dorsi and teres major) appears earlier and lasts longer than the rotator cuff [8]. The role of these muscles in the dynamic balance of the glenohumeral joint may be more important than usually thought, and their dysfunction should be taken into account when evaluating patients with SIS.

Travell and Simons reported that the symptoms produced by trigger points in the teres major muscle could be similar to one of other causes of pain in the shoulder, such as subacromial bursitis or supraspinatus tendonitis [9]. In fact, the area of referred pain associated with the trigger points 
of teres major is similar to the region where subjects diagnosed with pathology of the subacromial structures usually perceive pain [10].

In our daily clinical practice, we have frequently observed that patients diagnosed with SIS present myofascial trigger points in the teres major muscle, where palpation reproduces a pain that patients identify as their usual pain. However, only a few studies have analyzed the involvement of this muscle in the clinical context of SIS, and there seems to be no agreement on the role of the adductor muscles in the management of SIS. Some authors recommend that strengthening exercises of the adductor (due to their depressor moment arm) [11] and the rotator cuff muscles [12], should be included, while others recommend stretching the medial rotators (all of which are adductors) and isolated strengthening of the lateral rotators due to the fact that these muscles are fewer in number and weaker [13].

Our hypothesis is that teres major muscle involvement in the clinical status of patients diagnosed with SIS is greater than classically considered, and requires specific treatment. Our objective is to determine whether adding manual therapy specifically for the teres major muscle to a conventional physical therapy program produces better results than applying a conventional physical therapy program in isolation for patients with SIS.

\section{METHODOLOGY}

A randomized controlled experimental case series study was carried out. The participants were recruited at two centers: a Primary Care Center in the Spanish National Health System (Cornellà de Llobregat - Barcelona) and the FREMAP Mutual Society for Work-related Injuries and Occupational Illness (Arnedo - La Rioja). The IDIAP Jordi Gol Clinical Research Ethics Committee approved the protocol of this study on 2013-10-02, with code number $\mathrm{P} 13 / 082$. This study was registered with the US National Institutes of Health website: ClinicalTrials.gov identifier NCT02374073.

Due to the lack of previous studies with specific treatment of teres major muscle, there were no statistical data to estimate a previous calculation of the sample size. Participation in the study was offered to the patients at both centers who were referred for SIS treatment during the period from January to March 2014.
The inclusion criteria were: Age 18 years and over, a clinical diagnosis of SIS, the presence of myofascial trigger points in the teres major muscle, and signing of the informed consent. The Hawkins-Kennedy [14] and Neer [15] tests were used for the clinical diagnosis of SIS. This inclusion criteria was met if one (or both) of these tests were positive, i.e. if the patient's pain was reproduced. Trigger point localization in the teres major muscle was carried out with the patient in a supine position, with the shoulder in a nonpainful abduction position in the scapular plane and searching for the presence of nodules within a taut band with a digital pincer grip. It was considered positive if the patient showed some pain or signs of pain avoidance.

The exclusion criteria were: The presence of wounds or cutaneous alterations in the shoulder region, previous surgery on the shoulder, the presence of an acute inflammatory process in the shoulder ( $<7$ days), being involved in litigation or compensation processes, and not having a command of the language that could make the informed consent impossible to understand.

Fig. 1 shows the design of the study and the flow of the participants throughout each stage of the study, from the initial contact to the analysis of the results. Ninety-eight patients were asked to participate in this study and none refused to take part, but 40 were excluded. Of the 98 patients contacted, 86 showed positive results in the clinical tests for SIS and 12 did not. Of the 86 patients with a positive result in the clinical test for SIS, 60 presented trigger points in the teres major muscle, and 26 did not. Of the 60 people that met the inclusion criteria, 2 were excluded due to having pending litigation or compensation.

The patients recruited for this study $(n=58)$ were randomly assigned to one of the two groups: either the intervention group or the control group. Randomization was stratified for each center, and was carried out before subject recruitment with a computer program that generated a list of consecutive numbers which were assigned to one of the study groups.

During the treatment period, 8 subjects were lost from follow-up, 4 of which were in the intervention group and 4 in the control group, due to various personal reasons unrelated to the study. The treatment protocol was completed with 50 subjects ( 25 in each group) who joined the sample of this study. 


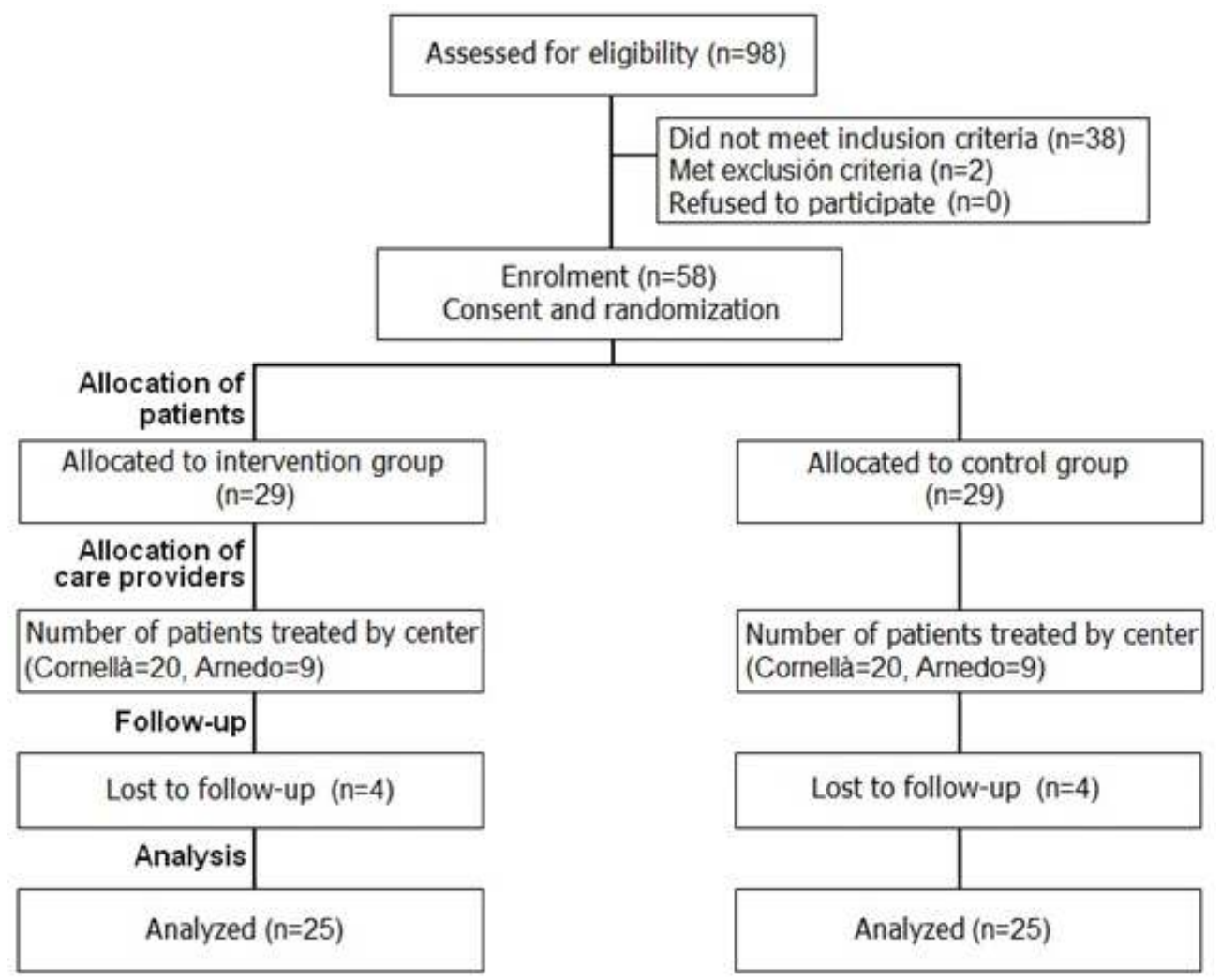

Fig. 1. Consort diagram. Participants flow throughout the study

Regardless of the assigned group, all participants received a three-week protocol of treatment, with daily sessions of therapeutic exercises (30 minutes) performed in non-painful arc of motion only under supervision by a physiotherapist, analgesic electrotherapy (20 minutes) and cryotherapy (10 minutes). The participants in the intervention group also received a functional massage in the teres major muscle.

Functional massage is a manual therapy technique, indicated in cases of painful muscle tightness [16] that combines a rhythmic and nonpainful passive joint mobilization in the direction of muscle stretching, together with compression/ decompression of the muscle to be treated [17]. It begins with compression of the muscle in a position of muscle shortening, and progresses with the passive mobilization of the joint in the direction of muscle stretching until the tightening reaches the compressed muscle area. The muscle compression is then removed and the joint is moved to the starting joint position and the procedure is repeated rhythmically (Fig. 2).

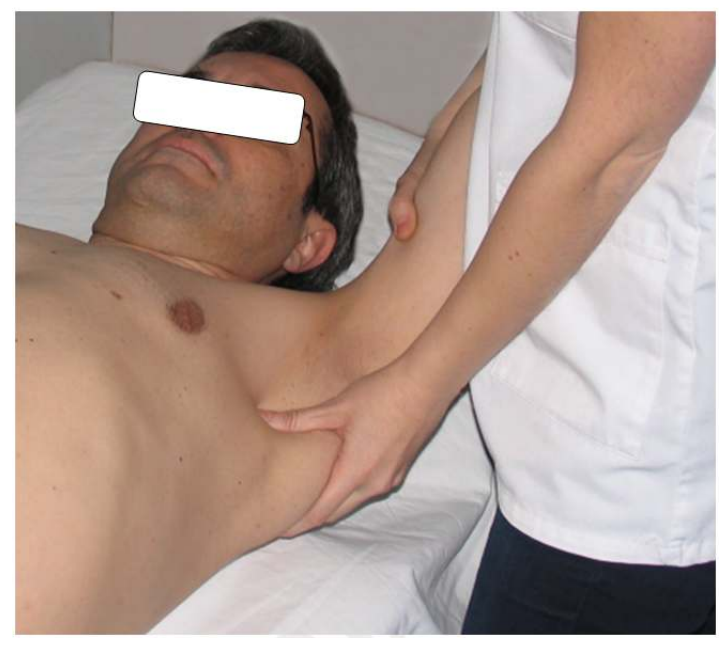

Fig. 2. Final position of the functional massage technique

The functional massage technique has some shared characteristics with the trigger point pressure release technique proposed by Travell and Simons as a substitute for the ischemic compression technique [18]. In the pressure 
release technique, non-painful maintained pressure is applied in a lengthening position of the muscle, while in the functional massage technique the pressure is applied intermittently. This reduces the likelihood of causing ischemia, and passive joint mobilization in the direction of muscle stretching may improve the local circulatory flow, thereby minimizing the energy crisis at the myofascial trigger points.

In our study, the functional massage technique was applied within 5 minutes of each treatment session, with a frequency of 20 to 25 movements per minute.

The following result variables were measured, immediately before and after the treatment period: pain intensity, level of function and active range of movement. The subjective opinion of the subject regarding the results obtained was also recorded at the end of the treatment period.

A Visual Analogue Scale (VAS) 100 millimeters in length without intermediate references was used to measure pain intensity [19]. The subjects were asked to register their level of pain in the shoulder region. If the patient felt that the pain intensity was variable, the subject was asked to register the pain intensity perceived in the shoulder region at the most painful point in time.

The level of function was measured with the simplified Constant-Murley Test, in which force measurement is not considered, with a potential maximum score of 75 points [20]. The use of the simplified test is justified because the force measurement is the less standardized parameter of the original test, with various procedures for registration (and scoring) that have not been validated. Moreover, the measurement position (abduction) may be painful for patients with SIS, hindering precise measurement [21].

The active range of movement in flexion, abduction, extension and lateral rotation was measured with a two-arm universal goniometer and the results were expressed in degrees. The flexion and extension were measured in the sagittal plane, with the elbow in extension and the forearm in the mid position of pronosupination (thumb pointing forward). Abduction was measured in the scapular plane with the elbow in extension and the forearm in the mid position of pronosupination. Lateral rotation was measured in neutral position of the shoulder (arm beside the trunk), elbow in $90^{\circ}$ of flexion and forearm in the mid position of pronosupination [22]. The active range of movement in medial rotation was measured with the hand-behind-back reach test. The position reached with the tip of the thumb was marked with a dermographic pencil, and the distance between this mark and the lower end of the spinous process of $\mathrm{C} 7$ was measured in centimeters; the shorter the distance, the greater the mobility [22].

The subjective results perceived by the subjects after the treatment were rated using a Global Rating of Change scale (GROC scale) [23].

The process of measurement and data collection and the treatment protocol were determined by the physical therapists at the two participating centers and practiced during a common training session.

Blinding techniques were not applied during this study. The same physical therapist that collected the variable data applied the manual treatment, and could not be blinded. The participants assigned to the control group were aware that no additional manual therapy was applied.

Statistical analysis of the results was carried out with version 20.0 of the SPSS program, using non-parametrical tests due to the reduced sample size. The level of significance was established at alpha $=.05$ and the limits of the confidence interval at $95 \%$. In order to compare the groups at the beginning of the study, the Chisquare and Fisher exact tests were used for the qualitative variables, and the Mann-Whitney $U$ test was used for the quantitative variables. The Wilcoxon signed rank test was used in order to analyze the intra-group differences in the result variables. ANCOVA was used for the comparison between groups.

To estimate the clinical relevance of the results, apart from the results from the GROC scale that were analyzed with the Fisher exact test, the effect size of the inter-group results were estimated (difference of standardized averages, Cohen's d) with an online calculator (http://www.uccs.edu/ /becker/). Cohen describes $0.2,0.5$, and 0.8 as a small, moderate and large effect size respectively [24].

\section{RESULTS AND DISCUSSION}

The average age of the participants was 61.6 years (SD 10.71) with a range between 23 and 80 years, $66 \%$ were women. The most affected 
shoulder was the right one (68\%). Only one participant was left-handed. The demographic characteristics of the participants, including the values of the result variables at baseline, are shown in Table 1.

No statistically significant between-group difference was found for any of the qualitative demographic variables. For the quantitative demographic variables, there were statistically significant between-group differences in age $(p=.02)$ and extension range of movement $(p=.01)$ at baseline. The differences in pain duration, pain intensity, function and the remaining mobility variables were not statistically significant.

Table 1. Baseline characteristics of the participants

\begin{tabular}{|c|c|c|}
\hline Variables & Intervention group $(\mathrm{n}=25)$ & Control group $(n=25)$ \\
\hline Age in years & $58.1(10.30)$ & $65.2(10.08)$ \\
\hline \multicolumn{3}{|l|}{ Sex } \\
\hline Male N (\%) & $11(44)$ & $6(24)$ \\
\hline Female N (\%) & $14(56)$ & $19(76)$ \\
\hline \multicolumn{3}{|l|}{ Affected shoulder } \\
\hline Right N (\%) & $18(72)$ & $16(64)$ \\
\hline Left N (\%) & $7(28)$ & $9(36)$ \\
\hline Pain duration in months & $13.16(13.64)$ & $10.64(11.38)$ \\
\hline \multicolumn{3}{|l|}{ Occupation (out-home) } \\
\hline Active N (\%) & $11(44)$ & $5(20)$ \\
\hline Unemployed N (\%) & $1(4)$ & $1(4)$ \\
\hline Retired N (\%) & $9(36)$ & $13(52)$ \\
\hline No $N(\%)$ & $4(16)$ & $6(24)$ \\
\hline \multicolumn{3}{|l|}{ Sporting activity } \\
\hline Yes N (\%) & $15(60)$ & $17(68)$ \\
\hline No $N(\%)$ & $10(40)$ & $8(32)$ \\
\hline \multicolumn{3}{|l|}{ Previous trauma } \\
\hline Si N (\%) & $2(8)$ & $7(28)$ \\
\hline No $\mathrm{N}(\%)$ & $23(92)$ & $18(72)$ \\
\hline \multicolumn{3}{|l|}{ Type of pain } \\
\hline Continuous N (\%) & $9(36)$ & $11(44)$ \\
\hline In specific movements $\mathrm{N}(\%)$ & $16(64)$ & $14(56)$ \\
\hline \multicolumn{3}{|l|}{ Predominant pain } \\
\hline Daytime pain N (\%) & $13(52)$ & $8(32)$ \\
\hline Nighttime pain N (\%) & $12(48)$ & $17(68)$ \\
\hline \multicolumn{3}{|l|}{ Most painful movement } \\
\hline Lying on the affected side N (\%) & $7(28)$ & $3(12)$ \\
\hline Lying on the non-affected side $\mathrm{N}(\%)$ & $1(4)$ & $1(4)$ \\
\hline Elevation N (\%) & $11(44)$ & $11(44)$ \\
\hline Hand to back N (\%) & $5(20)^{\prime}$ & $9(36)$ \\
\hline Others N (\%) & $1(4)$ & $1(4)$ \\
\hline \multicolumn{3}{|l|}{ Pharmacological treatment } \\
\hline Yes N (\%) & $14(56)$ & $11(44)$ \\
\hline No $\mathrm{N}(\%)$ & $11(44)$ & $14(56)$ \\
\hline Pain intensity (1) & $61.0(21.34)$ & $63.5(21,80)$ \\
\hline Function (Constant-Murley) & $41.4(12.85)$ & $45.6(9.92)$ \\
\hline Flexion (2) & $118.9(30.00)$ & $118.2(23.91)$ \\
\hline Abduction (2) & $111.6(28.27)$ & $116.4(22.05)$ \\
\hline Extension (2) & $41.9(16.97)$ & $29.6(9.77)$ \\
\hline Lateral rotation (2) & $29.8(17.24)$ & $25.6(13.21)$ \\
\hline Medial rotation (3) & $26.7(13.81)$ & $33.4(13.30)$ \\
\hline
\end{tabular}

(3) Distance in centimeters from spinous process of C7 to the tip of the thumb 
Intra-group analysis of the differences between baseline and post-treatment assessments are shown in Table 2. In the intervention group, all the result variables showed a statistically significant improvement. In the control group, the pain intensity and level of function variables had a statistically significant improvement, while no mobility variables had a statistically significant improvement.

In the between-groups comparison, the intervention group showed a larger improvement in all the result variables, except the similar result in both groups for pain intensity (Fig. 3). ANCOVA results, considering age and the initial values of each result variable as covariables, were statistically significant in abduction $(p=.01)$, extension $(p=.02)$ and lateral rotation $(p=.02)$ movements.

The clinical significance of the between-group differences, analyzed by estimating the effect size (Cohen's d) showed a small effect size $(.2$ to .5$)$ at the level of function, flexion, extension and lateral rotation; a moderate effect size $(=0.5)$ in abduction, and no significance for pain intensity and medial rotation. The subjective results expressed by the participants using a GROC scale are shown in Table 3 and are very similar for both groups.

Table 2. Changes in each variable between baseline and post-treatment assessments

\begin{tabular}{lllllll}
\hline Variable & \multicolumn{3}{c}{ Intervention group } & \multicolumn{3}{c}{ Control group } \\
\cline { 2 - 7 } & Mean (SD) & Cl 95\% & P & Mean (SD) & Cl 95\% & p \\
\hline Pain intensity (1) & $21.16(19.16)$ & $13.2 / 29.1$ & .01 & $22.92(20.90)$ & $14.3 / 31.5$ & .01 \\
Function (C-M) & $10.60(8.36)$ & $7.1 / 14.1$ & .01 & $6.92(7.75)$ & $3.7 / 10.1$ & .01 \\
Flexion (2) & $14.76(17.24)$ & $7.6 / 21.9$ & .01 & $4.48(19.08)$ & $-3.4 / 12.4$ & n.s. \\
Abduction (2) & $23.00(15.93)$ & $16.4 / 29.6$ & .01 & $1.00(21.45)$ & $-7.9 / 9.9$ & n.s. \\
Extension (2) & $5.64(9.50)$ & $1.7 / 9.6$ & .01 & $0.84(7.85)$ & $-2.4 / 4.1$ & n.s. \\
Lateral rotation (2) & $8.76(10.53)$ & $4.4 / 13.1$ & .01 & $0.72(8.21)$ & $-2.7 / 4.1$ & n.s. \\
Medial rotation (3) & $2.60(4.54)$ & $0.7 / 4.5$ & .01 & $1.56(6.10)$ & $-1.0 / 4.1$ & n.s. \\
\hline
\end{tabular}

Note. p: value of the intra-group comparison. n.s. not significant. C-M: Constant-Murley. (1) VAS in millimeters from 0 to 100. (2) Mobility in degrees from zero to maximum active range of motion. (3) C7-thumb distance in centimeters

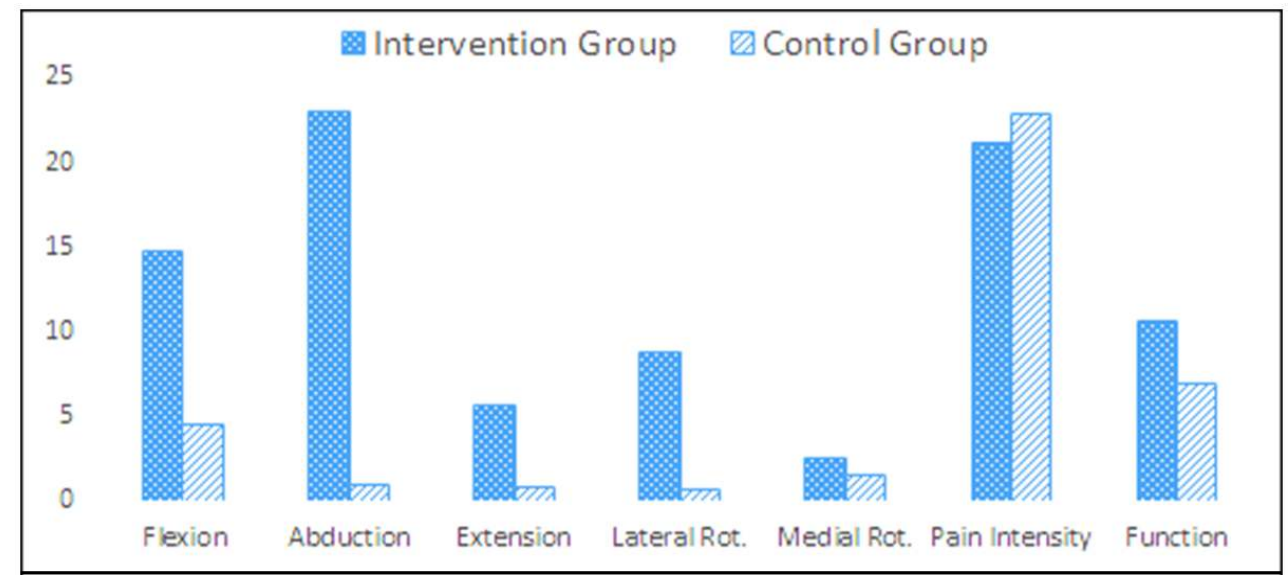

Fig. 3. Graphical representation of changes on each variable

Table 3. Results of the Global Rating of Change scale (GROC scale)

\begin{tabular}{lll}
\hline & Intervention group & Control group \\
\hline Clinical improvement (1) & 17 & 16 \\
Without clinical changes (2) & 8 & 8 \\
Clinical worsening (3) & 0 & 1 \\
\hline \multicolumn{2}{c}{ Note: (1) Values between "Moderately better" and "A very great deal better". (2) Values between "Somewhat } \\
better" and "Somewhat worse". (3) Values between "Moderately worse" and "A very great deal worse"
\end{tabular}


The results of this study support our hypothesis that the teres major muscle is also involved in the clinical status of many patients diagnosed with SIS, and that adding a specific treatment helps to obtain better results than a conventional physical therapy treatment.

The teres major muscle had myofascial trigger points in sixty $(70 \%)$ of the 86 patients showing positive results in clinical tests for SIS, which is similar to the results of Bron et al. [25] who concluded that $76 \%$ of the subjects with pain in the shoulder with a non-traumatic etiology had trigger points ( $27 \%$ active and $49 \%$ latent) in the teres major muscle.

Although conventional physical therapy has enabled us to achieve satisfactory results for these patients with improvements in pain intensity and level of function, the addition of manual therapy focused on the trigger points in the teres major muscle improved the mobility results, and achieved statistical significance in abduction, extension and lateral rotation, as well as clinical significance at the level of function, abduction, extension and lateral rotation. Other studies showed similar results. The systematic review of Kung JE concluded that therapeutic exercises are effective for improving pain and function, but not for the range of movement or the force of the subjects with SIS, and that its efficacy improves if manual therapy is added [26].

Pain provocation in the structures of the subacromial space of previously asymptomatic subjects alters the pattern of muscle activation, thereby increasing the activity of the adductor muscles [27]. It has also been shown that patients with a full-thickness tear of the rotator cuff present an increased activation of the deltoid muscle, considered to compensate for the absence of the supraspinatus, together with an increase in the activity of the teres major and latissimus dorsi [28]. This increased activation of the adductor muscles is attributed to the need to stabilize the humeral head in order to minimize the impingement and protect the subacromial structures. Despite the almost complete pain alleviation (from 7.7 to 0.9 in VAS) due to lidocaine subacromial infiltration, it did not recover the pattern of activation considered normal in the overall sample, but only partially and only in some subjects [28]. In our study, a conventional therapeutic approach focused on the subacromial structures, despite achieving a significant reduction in the pain intensity regardless of the group assignation, it did not enable a recovery of mobility unless specific treatment of the dysfunctional muscle was added, in this case, after functional massage treatment of the myofascial trigger points in the teres major muscle. Studies of the effects of the pressure release technique also show an increase in the restricted mobility of the muscles involved $[29,30]$.

Although the intervention group showed better results for all variables of mobility, function improvement measured with the simplified Constant-Murley Test was only slightly higher than that obtained by the control group. The Constant-Murley test is an aggregated score of various items, including four shoulder movements, but only two movements (flexion and abduction) are rated using the angular range of motion, and the score only increases with every 30 degrees of improvement. Minor improvements, albeit statistically and clinically significant, cannot be reflected in the global score.

The subjective results expressed by the participants using a GROC scale were very similar for both groups, as well as the improvement in pain intensity and function. Therapeutic exercises are effective for improving pain and function [26] and adding a specific manual technique for the teres major muscle does not have any significant additional effect on these variables. Although it is plausible to consider that pain and function are the most important items to support the subjective opinion of the patient, we found no studies of the relationship between the results of the GROC scale and other clinical variables in patients with subacromial impingement syndrome.

Our study supports the existing evidence, which revealed that in the treatment of the pathology of the subacromial space, a therapeutic approach of physical therapy that includes manual therapy techniques is superior to a physical therapy approach that does not include those techniques $[31,32]$. Choosing the manual technique to be applied to the specifically affected structures may improve the results in these patients.

Our study presents some limitations, such as the lack of blinding of the evaluator and the reduced sample size. We were also unable to ensure the representativeness of our sample, so we cannot guarantee that the data obtained have external validity. Additionally, we must take into account 
that a potential placebo effect has not been controlled, and this may have an influence on the subjects treated with an additional manual therapy technique.

\section{CONCLUSION}

Although our conclusions must be considered with caution due to the limitations of our study, our results show that the association between SIS and trigger points in the teres major muscle may be more frequent than described in the literature, and adding functional massage of the teres major muscle helps to achieve better results in the glenohumeral range of movement.

\section{CONSENT}

All authors declare that written informed consent was obtained from all the patients for publication of this article and the accompanying images.

\section{ETHICAL APPROVAL}

All authors hereby declare that the protocol of this study was examined and approved by the IDIAP Jordi Gol Clinical Research Ethics Committee on 2013-10-02, with code number $\mathrm{P} 13 / 082$. All the experiments have therefore been performed in accordance with the ethical standards laid down in the 1964 Declaration of Helsinki.

\section{COMPETING INTERESTS}

Authors have declared that no competing interests exist.

\section{REFERENCES}

1. Urwin M, Symmons D, Allison T, Brammah $\mathrm{T}$, Busby $\mathrm{H}$, Roxby M, et al. Estimating the burden of musculoskeletal disorders in the community: The comparative prevalence of symptoms at different anatomical sites, and the relation to social deprivation. Ann Rheum Dis. 1998;57(11):649-55.

2. Chard MD, Hazleman R, Hazleman BL, King $\mathrm{RH}$, Reiss BB. Shoulder disorders in the elderly: A community survey. Arthritis Rheum. 1991;34(6):766-9.

3. VII Encuesta Nacional de Condiciones del Trabajo. Instituto Nacional de Seguridad e Higiene en el Trabajo. Ministerio de Empleo y Seguridad Social.

(Accessed 31 August 2012)

Available; http://encuestasnacionales.oect. $\underline{\text { es }}$
4. van der Windt DA, Koes BW, de Jong BA, Bouter LM. Shoulder disorders in general practice: Incidence, patient characteristics, and management. Ann Rheum Dis. 1995; 54(12):959-64.

5. MacDermid JC, Ramos J, Drosdowech D, Faber K, Patterson S. The impact of rotator cuff pathology on isometric and isokinetic strength, function, and quality of life. J Shoulder Elbow Surg. 2004;13(6):593-8.

6. Diederichsen LP, Nørregaard J, DyhrePoulsen P, Winther A, Tufekovic G, Bandholm $\mathrm{T}$, et al. The activity pattern of shoulder muscles in subjects with and without subacromial impingement. J Electromyogr Kinesiol. 2009;19(5):789-99.

7. Inman VT, Saunders JB, Abbott LC. Observations on the function of the shoulder joint. $\mathrm{J}$ Bone Joint Surg Am. 1944;26:1-30.

8. Hawkes $\mathrm{DH}$, Alizadehkhaiyat $\mathrm{O}$, Fisher AC, Kemp GJ, Roebuck MM, Frostick SP. Normal shoulder muscular activation and co-ordination during a shoulder elevation task based on activities of daily living: An electromyographic study. J Orthop Res. 2012;30(1):53-60.

9. Simons DG, Travell JG, Simons LS. Dolor y disfunción miofascial. El manual de los puntos gatillo. Volumen 1. Mitad superior del cuerpo. 2a ed. Madrid: Editorial Médica Panamericana. 2002;727.

10. Gerber C, Galantay RV, Hersche O. The pattern of pain produced by irritation of the acromioclavicular joint and the subacromial space. J Shoulder Elbow Surg. 1998;7(4):352-5.

11. Halder AM, Zhao KD, Odriscoll SW, Morrey BF, An KN. Dynamic contributions to superior shoulder stability. J Orthop Res. 2001;19(2):206-12.

12. Sharkey NA, Marder RA. The rotator cuff opposes superior translation of the humeral head. Am J Sports Med. 1995; 23(3):270-5.

13. Marc T, Gaudin T, Teissier J. Bases biomécaniques de la rééducation des tendinopathies de la coiffe des rotateurs. KS. 2008;489:5-9. French.

14. Hawkins RJ, Kennedy JC. Impingement syndrome in athletes. Am J Sports Med. 1980;8(3):151-8.

15. Neer CS. Impingement lesions. Clin Orthop. 1983;173:70-7.

16. Wolf U. Angewandte Manuelle Therapie. Band 1: Halswirbelsäule - Kiefergelenk - 
Schulter - Ellenbogen - Hand. MunchenJena: Ed. Urban \& Fischer; 2001.

17. Tricás JM. Cuadernillos Prácticos de Fisioterapia, Masaje Funcional. Zaragoza: Fundación Empresa Universidad de Zaragoza. 2001;4.

18. Simons DG, Travell JG, Simons LS. Dolor y disfunción miofascial. El manual de los puntos gatillo. Volumen 1. Mitad superior del cuerpo. $2^{\mathrm{a}}$ ed. Madrid: Editorial Médica Panamericana. 2002;174-5.

19. Huskisson EC. Measurement of pain. Lancet. 1974;2(7889):1127-31.

20. Patel VR, Singh D, Calvert PT, Bayley JIL. Arthroscopic subacromial decompression: Results and factors affecting outcome. $J$ Shoulder Elbow Surg. 1999;8(3):231-7.

21. Barra-López ME. El test de ConstantMurley. Una revisión de sus características. Rehabilitación (Madr). 2007;41(5):228-35. Spanish.

22. Greene WB, Heckman JD. The clinical measurement of joint motion. American Academy of Orthopaedic Surgeons; 1994.

23. Stratford PW, Binkley FM, Riddle DL. Health status measures: Strategies and analytic methods for assessing change scores. Phys Ther. 1996;76(10):1109-23.

24. Cohen J. The concepts of power analysis. In: Cohen J, Editor. Statistical power analysis for the behavioral sciences. Hillsdale, New Jersey: Academic Press Inc. 1988;1-17.

25. Bron C, Dommerholt J, Stegenga B, Wensing $M$, Oostendorp RA. High prevalence of shoulder girdle muscles with myofascial trigger points in patients with shoulder pain. BMC Musculoskelet Disord. 2011;12:139.

26. Kuhn JE. Exercise in the treatment of rotator cuff impingement: A systematic review and a synthesized evidence-based rehabilitation protocol. J Shoulder Elbow Surg. 2009;18(1):138-60.

27. Diederichsen LP, Winther A, DyhrePoulsen P, Krogsgaard MR, Nørregaard J. The influence of experimentally induced pain on shoulder muscle activity. Exp Brain Res. 2009;194(3):329-37.

28. Steenbrink $F$, de Groot JH, Veeger HE, Meskers CG, van de Sande MA, Rozing PM. Pathological muscle activation patterns in patients with massive rotator cuff tears, with and without subacromial anaesthetics. Man Ther. 2006;11(3):231-7.

29. Aguilera FJ, Martín DP, Masanet RA, Botella AC, Soler LB, Morell FB. Immediate effect of ultrasound and ischemic compression techniques for the treatment of trapezius latent myofascial trigger points in healthy subjects: A randomized controlled study. J Manipulative Physiol Ther. 2009;32(7):51520.

30. Oliveira-Campelo NM, de Melo CA, Alburquerque-Sendín F, Machado JP. Short- and medium-term effects of manual therapy on cervical active range of motion and pressure pain sensitivity in latent myofascial pain of the upper trapezius muscle: A randomized controlled trial. J Manipulative Physiol Ther. 2013;36(5):3009.

31. Senbursa G, Baltaci G, Atay A. Comparison of conservative treatment with and without manual physical therapy for patients with shoulder impingement syndrome: A prospective, randomized clinical trial. Knee Surg Sports Traumatol Arthrosc. 2007;15(7):915-21.

32. Białoszewski D, Zaborowski G. Usefulness of manual therapy in the rehabilitation of patients with chronic rotator cuff injuries. Preliminary report. Ortop Traumatol Rehabil. 2011;13(1):9-20.

(c) 2016 Barra-López et al.; This is an Open Access article distributed under the terms of the Creative Commons Attribution License (http://creativecommons.org/licenses/by/4.0), which permits unrestricted use, distribution, and reproduction in any medium, provided the original work is properly cited.

Peer-review history:

The peer review history for this paper can be accessed here: http://sciencedomain.org/review-history/16572 